\title{
Nonadiabatic interaction between a charged particle and an MHD pulse
}

\author{
Y. Kuramitsu ${ }^{1}$ and T. Hada ${ }^{2}$ \\ ${ }^{1}$ Institute of Laser Engineering, Osaka University, Suita, Japan \\ ${ }^{2}$ ESST, Interdisciplinary Graduate School of Engineering Sciences, Kyushu University, Kasuga, Japan
}

Received: 4 December 2007 - Revised: 13 February 2008 - Accepted: 13 February 2008 - Published: 17 March 2008

\begin{abstract}
Interaction between a magnetohydrodynamic (MHD) pulse and a charged particle is discussed both numerically and theoretically. Charged particles can be accelerated efficiently in the presence of spatially correlated MHD waves, such as short large amplitude magnetic structures, by successive mirror reflection (Fermi process). In order to understand this process, we study the reflection probability of particles by the MHD pulses, focusing on the adiabaticity on the particle motion. When the particle velocity is small (adiabatic regime), the probability that the particle is reflected by the MHD pulse is essentially determined only by the pitch angle, independent from the velocity. On the other hand, in the non-adiabatic regime, the reflection probability is inversely proportional to the square root of the normalized velocity. We discuss our numerical as well as analytical results of the interaction process with various pulse amplitude, pulse shape, and the pulse winding number. The reflection probability is universally represented as a power law function independent from above pulse properties.
\end{abstract}

\section{Introduction}

Since the space plasma is collisionless, various kinds of transport and dissipations take place via wave-particle interactions. In the acceleration of energetic particles or cosmic rays, magnetohydrodynamic (MHD) waves, which are basic low frequency waves in plasmas, play an essential role. Diffusion in the velocity space (energy diffusion), transport in the real space, and the pitch angle diffusion of the particles by the MHD waves have been discussed extensively, however, most of the arguments are based on the quasi-linear theory (QL) (e.g., Kennel and Engelmann, 1966; Lee, 1971; Lyons, 1974; Gary and Feldman, 1978; Lee, 1982; Schlickeiser, 1989). There are two as-

Correspondence to: Y. Kuramitsu

(kuramitsu-y@ile.osaka-u.ac.jp) sumptions in the quasi-linear theory; the amplitude of the wave is small, and the random phase approximation (R.P.A), i.e., there is no correlation between wave modes. However, the MHD waves observed in space often have large amplitude, therefore, it is expected to have phase correlation via nonlinear interaction between wave modes (Nariyuki and Hada, 2005, 2006). In fact, large amplitude MHD waves with pulse like structures are abundant in space plasmas; for instance, short large amplitude magnetic structures (SLAMS) (Schwartz and Burgess, 1991; Schwartz et al., 1992; Giacalone et al., 1993; Mann et al., 1994) and shocklets (Hoppe et al., 1981; Tsurutani et al., 1990, 1997). These waveforms are considered as an evidence for strong spatial phase correlation of wave modes (Koga and Hada, 2003; Hada et al., 2003). For acceleration and thermalization of charged particles by these waves, the assumptions of the quasi-linear theory are not appropriate.

Kuramitsu and Hada (2000) reported the effects of the wave large amplitude and of spatial correlation of wave modes using test particle simulations. When the waves have large amplitude, the energy diffusion coefficients from the numerical experiments are larger than those of the quasilinear prediction. When the waves have strong spatial correlation, not only the statistics or the energy diffusion coefficients but the scattering mechanism of particles is also greatly different from the predictions by the quasi-linear theory. In contrast to the random fields where the particles perform resonance scattering continuously resulting in diffusion in the velocity space, in the presence of waves with strong spatial correlation, or solitary wave packets, the particles are accelerated by successive mirror reflection due to the propagating of the wave packets to the opposite directions each other. Mann and Classen (1995) and Classen and Mann (1998) discussed particle acceleration by SLAMS due to mirror reflections, however, their model depends on adiabatic theory. In Kuramitsu and Hada (2000) the particles are non-adiabatically reflected by the spatial phase correlated waves resunlting in efficient acceleration. In order to explain

Published by Copernicus Publications on behalf of the European Geosciences Union and the American Geophysical Union. 


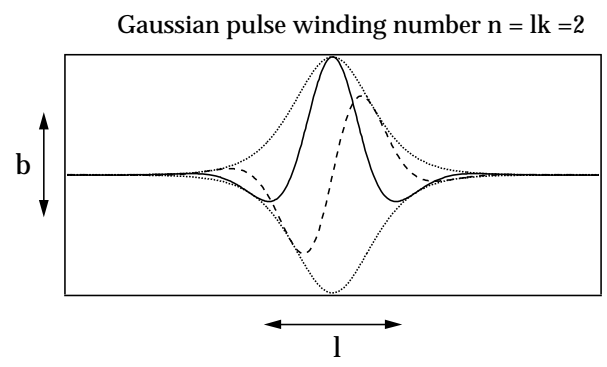

Fig. 1. A typical Gaussian pulse used in the run. The solid, broken, and dotted lines represent the $B_{y}, B_{z}$, and the envelope of wave packet.

the acceleration and scattering mechanism due to the MHD pulses, we discuss the mirror reflection of a particle by one wave packet, paying special attention to adiabatic and nonadibatic motion of charged particles.

In this paper we discuss nonlinear interaction between a charged particle and a wave packet numerically and theoretically. In Sect. 2 we describe our model and formulations. We numerically solve the particle trajectories, firstly, when the winding number equals zero, and then when it is finite. We calculate phase integrated and phase-pitch angle integrated reflection probabilities. In Sect. 3 we analyze the particle motion and formulate the reflection probability functions. We give conclusions in Sect. 4.

\section{Numerical experiment}

\subsection{Model and basic equations}

Quite often the mirror reflection of a particle is simply explained by the adiabatic invariant of a magnetic moment $m v_{\perp}^{2} /(2 B)$, where $m$ is the mass of the particle, $v_{\perp}$ is the perpendicular component of the particle velocity to the magnetic field, and $B$ is the strength of the magnetic field. Introducing the pitch angle of particle, $v_{\perp} \equiv V \sin \alpha$, with adiabatic interaction $V \sim$ const., one can write the invariance of the moment as

$\frac{\sin ^{2} \alpha}{B}=\frac{1}{B_{m}}$,

where $B_{m}$ is the magnetic field where the particle is reflected $(\alpha \rightarrow \pi / 2)$. Giving a wave packet with its maximum magnetic field strength $B_{\max }$, we define a critical pitch angle $\alpha_{c}$ by $\sin ^{2} \alpha_{c}=B_{0} / B_{\max }$, where $B_{0}$ is the background magnetic field. Particles with pitch angle less (larger) than $\alpha_{c}$ are transmitted to (reflected by) the wave packet. There is no energy dependence in this argument. If the particle distri- bution is isotropic, the reflection probability is given by the integration of Eq. (1) as,

$P_{a d}=\left(1-\frac{B_{0}}{B_{\max }}\right)^{1 / 2}$.

In reality, the interaction between a particle and a wave packet is not necessary to be adiabatic. For instance, SLAMS are considered to form a transition of the quasi-parallel shocks (Schwartz and Burgess, 1991), where energetic particles are often detected (Giacalone et al., 1993). A typical spatial scale of SLAMS is $\sim 3 \times 10^{6} \mathrm{~m}$, which is comparable to the gyroradius $\left(4 \times 10^{6} \mathrm{~m}\right)$ of the energetic particles with $\sim 20 \mathrm{keV}$ when the magnetic field magnitude is $\sim 5 \mathrm{nT}$ (Schwartz et al., 1992; Giacalone et al., 1993). Furthermore, the typical gradients of magnetic field changes within the SLAMS can be $\sim 10^{5} \mathrm{~m}$, much shorter than the SLAMS scale (Lucek et al., 2004). As far as energetic particles or cosmic rays are concerned, the adiabatic assumption is not appropriate. We consider neither an electrostatic potential of the wave packet, nor the temporal and spatial change of the wave phase or group velocity since the scale of the background change is much larger than that of pule-particle interactions. Note that when the group velocity of the wave packet is different from the phase velocity of local MHD waves (e.g., Schwartz et al., 1992) and/or when the electrostatic potential is concerned, there is a possibility of efficient particle acceleration by gyroresonant surfing (Kuramitsu and Krasnoselskikh, 2005a,b,c). It will be investigated elsewhere.

We numerically calculate a trajectory of a particle using a simple wave packet and discuss how a particle is reflected by the wave packet. We consider the interaction in a coordinate moving with the wave packet, where there is no wave electric field. Therefore, the particle energy is conserved. We solve an equation of motion of a particle,

$m \frac{d \boldsymbol{v}}{d t}=\frac{e}{c} \boldsymbol{v} \times \boldsymbol{B}$.

The D.C. magnetic field is in the $x$ direction and a Gaussian wave packet is given by (Fig. 1),

$\delta B=B_{y}(x)+i B_{z}(x)=A \exp \left[-\left(\frac{x}{L}\right)^{2}+i k x+i \theta\right]$

Here $A$ and $L$ are the amplitude and width of wave packet, and $k$ and $\theta$ are the wave number and phase constant of the carrier wave, respectively. Using the advantage of the constant particle energy, we normalize all the quantities using the magnitude of particle velocity $V$, the background magnetic field $B_{0}$, and the gyrofrequency $\Omega \equiv e B_{0} / m c$. We also define the normalized wave amplitude $b=A / B_{0}$, the normalized wave packet width $l=L \Omega / V=\zeta^{-1}$, the difference between particle gyrophase and a wave phase 
(a)

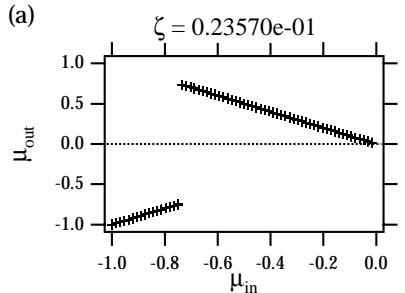

(b)

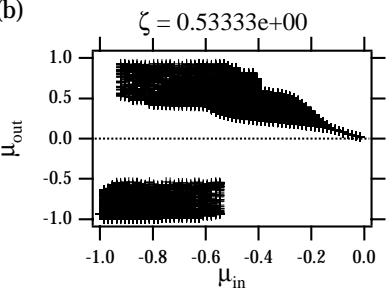

(c)

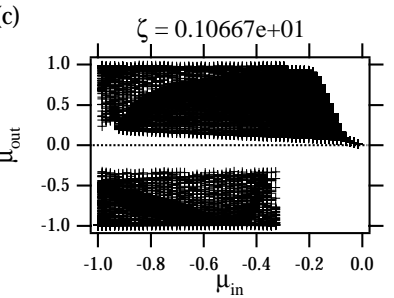

(d)

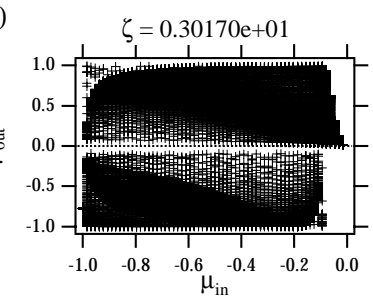

(e)

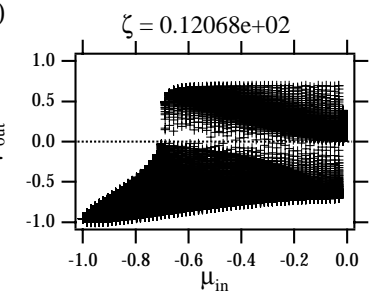

(f)

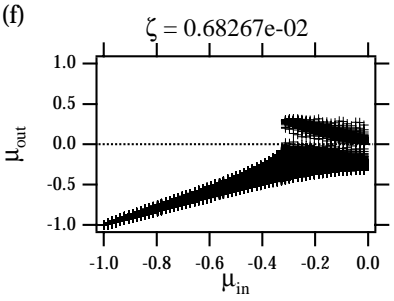

Fig. 2. Comparison between the particle pitch angle cosines before and after the interaction with the Gaussian pulse with $b=2$ and $n=0$. Each plot corresponds to different values of the normalized particle velocity, $V / L \Omega=\zeta$.

$\psi=\arctan B_{y} / B_{z}-\arctan v_{y} / v_{z}$, and the pitch angle cosine of the particle $\mu=\cos \alpha$. The normalized wave packet width $l$ (also the inverse value of it $\zeta$ ) has significant physical meaning. This is a ratio between the time necessary for a particle to traverse the wave packet and the Larmor period, or the ratio of Larmor radius to the scale of the width of wave packet. When $l$ is large, i.e., the wave packet width is large or equivalently the particle velocity is small. In this case the particle feels the change of the field slowly. In contrast, when $l$ is small, the particle feels a rapid change of the field and the interaction becomes non-adiabatic.

\subsection{Winding number $n=0$}

First let the "winding number" $n \equiv L k=0$. The winding number corresponds to the approximate number of rotations that the transverse magnetic field makes within the wave packet. We fix the amplitude of wave packet $(b=2)$ and change $l$. Thus the critical pitch angle cosine $\mu_{c} \equiv \cos \alpha_{c}=0.743$ from Eq. (1). We inject particles with various pitch angles and gyrophases from a point sufficiently distant from the wave packet.

Figure 2 shows the comparison of pitch angle cosine before and after the interaction. Different panels correspond to different values of $l$. Lines $\mu_{i n}= \pm \mu_{\text {out }}$ correspond to complete conservation of the first adiabatic invariant. When $\mu$
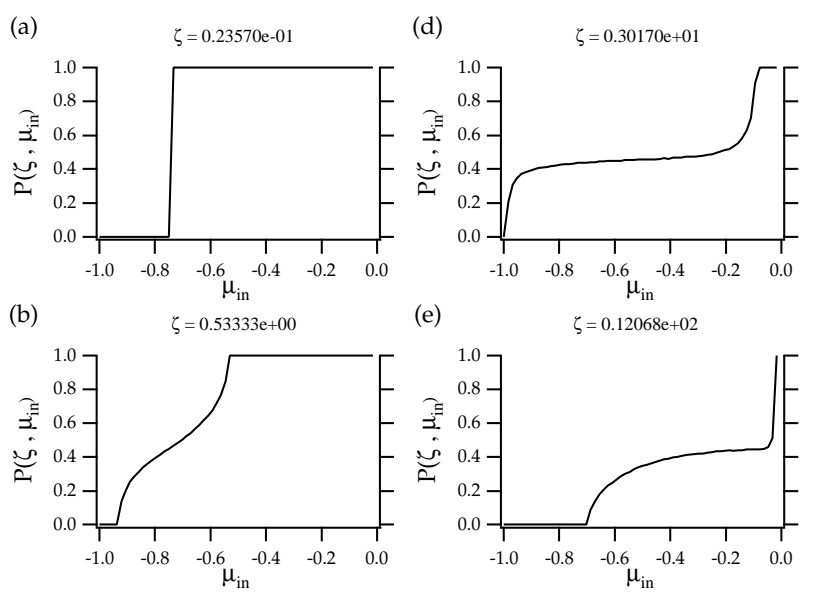

(e) $\quad \zeta=0.12068 \mathrm{e}+02$
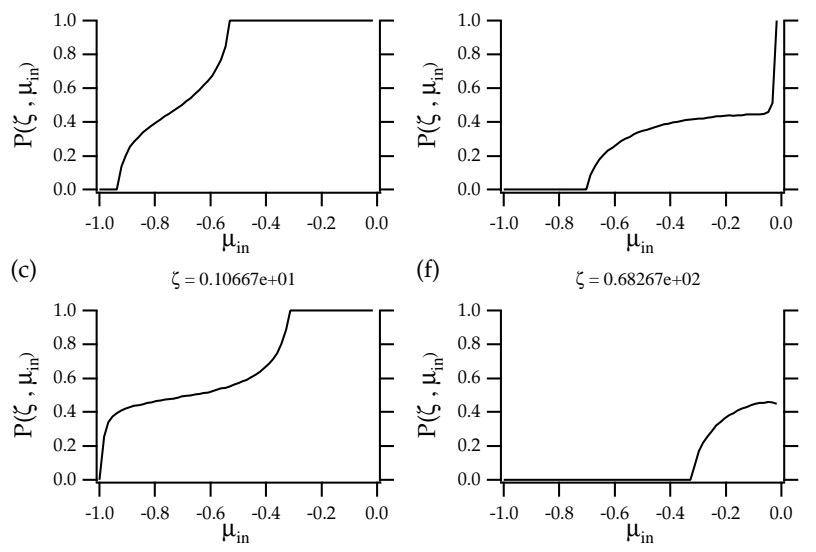

(f)

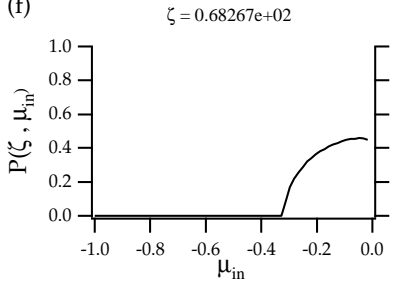

Fig. 3. Phase integrated reflection probability, $P\left(\zeta, \mu_{i n}\right)$, calculated from Fig. 2 is shown.

change its sign, the particle is reflected by the wave packet. On the other hand, when $\mu$ keep its sign same, the particle goes through the wave packet. When the winding number $n=0$ (linear polarization), it is not affected by whether the particles are injected from either side of the packet because of the symmetry. We release particles from the positive $x$ side of the packet, thus the particles with $\mu_{\text {out }}>0\left(\mu_{\text {out }}<0\right)$ correspond to reflected (transmitted) particles. When $l$ is large (i.e., small $\zeta \equiv l^{-1}$ ), the interaction is well explained by the adiabatic theory (a). The particles with $\mu<\mu_{c}$ (in loss cone) are transmitted to the wave packet, otherwise reflected, independent of $\psi$. When $l$ is slightly less, the breakdown of the adiabatic invariant is apparent; some particles in loss cone are reflected depending on $\psi$, and some particles outside the loss cone are transmitted (b). When $l$ is even less, it is difficult to find a trace of adiabatic interaction (c-e). With further reducing $l$, particles are scarcely reflected (e-f).

Figure 3 shows the phase-averaged reflection probability against $\mu$, obtained by phase integration of the results of Fig. 2. The panel (a) represents the adiabatic case; the reflection probability is 0 within the loss cone and 1 outside. As $\zeta$ increases, the probability becomes finite inside the loss cone (b). As $\zeta$ increases, the boundary between the transmittion and reflection becomes obscure $(c-d)$. With further increasing $\zeta, P$ continues to decrease and vanishes eventually.

Figure 4a summarizes the reflection probability in one figure. The vertical axis denotes $\zeta$ in a logarithmic scale, and a 

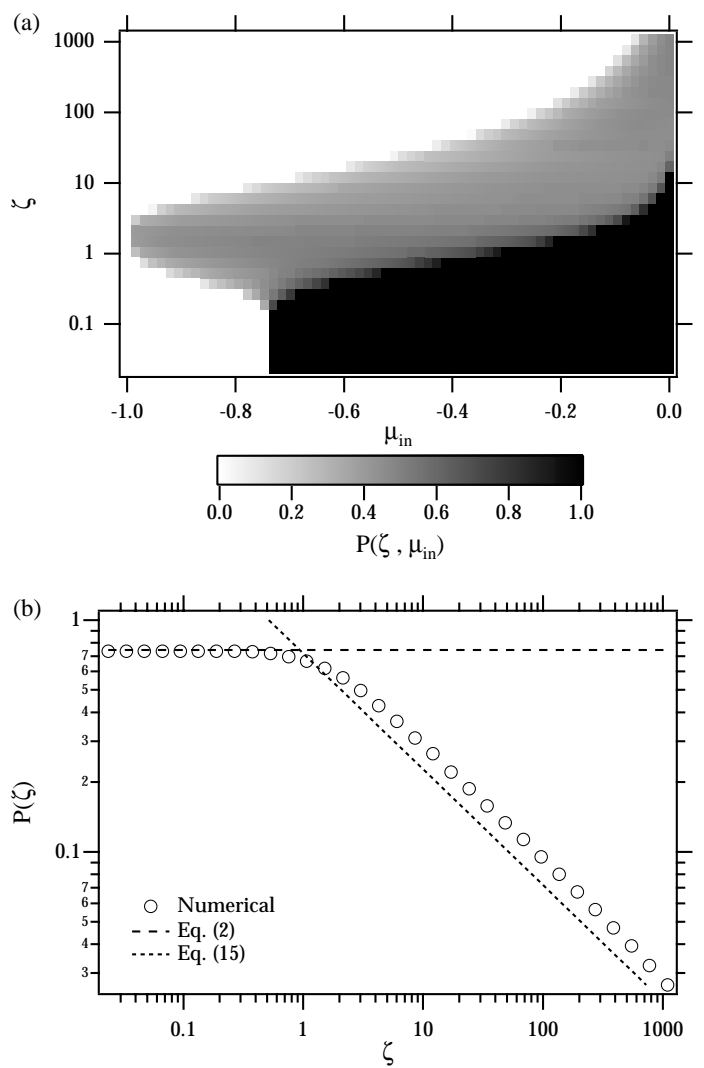

Fig. 4. In (a) image plot of the reflection probability $P\left(\zeta, \mu_{i n}\right)$ is shown with the same pulse parameters as in Fig. 2. In (b) pitchangle integrated reflection probability $P(\zeta)$ is shown. Values obtained from the numerical calculations are superposed in the plot as circles. Dashed and dotted lines denote the adiabatic theory Eq. (2), and the non-adiabatic asymptote Eq. (15), respectively.

horizontal axis is $\mu_{i n}$. It is clear that, when $\zeta$ is small, the interaction is adiabatic, while the non-zero values of reflection probability is particular enhanced around $\zeta \sim 1$. When $\zeta \gg 1$, $P$ decreases as $\left|\mu_{i n}\right|$ increases.

Further integration of $P\left(\zeta, \mu_{i n}\right)$ in Fig. 4a over $\mu_{i n}$ yields the reflection probability based on the isotopic distribution, $P(\zeta)$ in Fig. 4b. Here $P(\zeta)$ is plotted versus $\zeta$ in logarithmic scales. If $\zeta$ is small, the reflection probability is well explained by the adiabatic theory (Eq. 2). Non-adiabaticity appears around $\zeta \sim 1$. As $\zeta$ increases, the reflection probability decreases as $\zeta^{-1 / 2}$.

We show another example with $n=0$. Figure 5a shows the reflection probability $P\left(\zeta, \mu_{i n}\right)$ with the wave packet amplitude $b=6$, and Fig. $5 \mathrm{~b}$ shows its integration over $\mu_{i n}$. Note that the reflection probability is enhanced at $\zeta \sim 1$ with $\mu_{i n} \sim-1$ (beam particles) in (a). This enhancement was not found when $b=2$, although Fig. 5b shows similar characteristics to the case with $b=2$; the curve consists of an adiabatic part and non-adiabatic power law decrease. The index of power law is again $-1 / 2$.
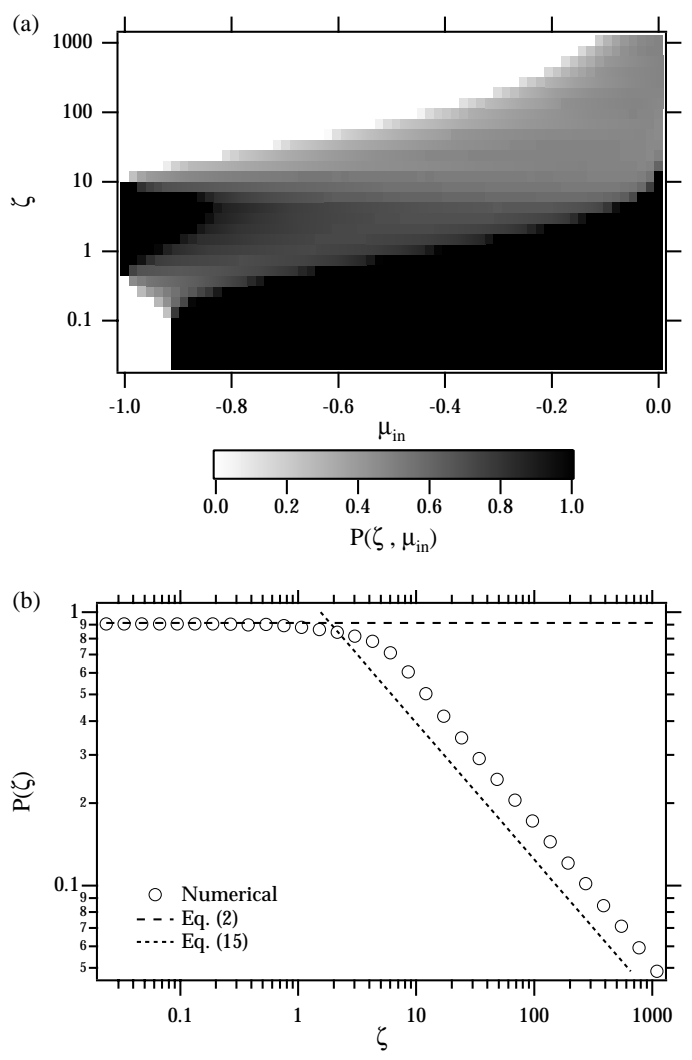

Fig. 5. Same as Fig. 4 except that the pulse amplitude is $b=6$.

\subsection{Winding number $n \neq 0$}

Here we discuss non-zero winding number (we chose $n=2$ with amplitude $b=2$ ). In this case the particles with $v_{\|}<0$ can resonate with the wave, while the particles with $v_{\|}>0$ cannot. Note that we consider particles with positive charge here, however, the resonance condition also depends on the sign of the particle charge. When negative charged particles are concerned, one has to simply change the sign of the resonance condition. Figures 6 and 9 show the results plotted in the same formats as Figs. 2 and $4 \mathrm{~b}$. The only difference in the parameter is $n=2$. In Fig. 6a, the interaction between the wave packet and the particle can be well explained by the adiabatic theory. The interaction does not depend on from which side the particle collides the wave packet. As $\zeta$ increases (b-e), the scattering shows some deviation from the adiabatic line as already shown in Fig. 2, and moreover the right and left symmetry is broken. In particular, the deviation from the adiabatic line $\left(\mu_{\text {in }}= \pm \mu_{\text {out }}\right)$ is larger for the particles with resonant sense $\left(\mu_{i n}<0\right)$ than the others. The scattering of particles becomes symmetric with respect to $\mu_{i n}$ in the regime $\zeta \gg 1(\mathrm{e}-\mathrm{f})$.

Figure 7 shows the phase integrated reflection probability calculated from Fig. 6. We confirm that there is a breakdown of the adiabaticity and the symmetry. The reflection probability is complex, especially for $\mu_{i n}<0$. 
(a)

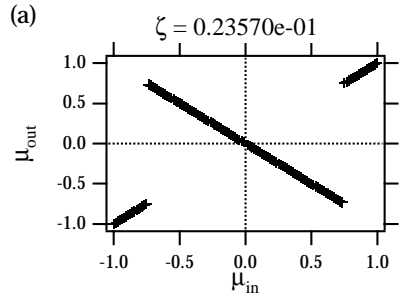

(b)

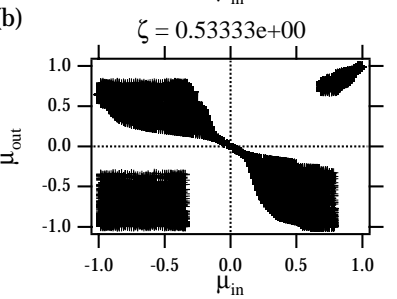

(c)

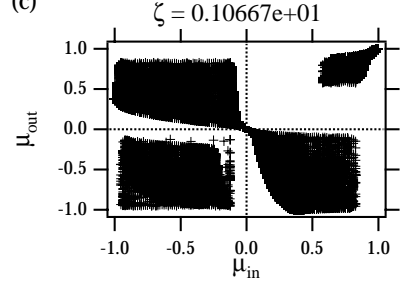

(d)

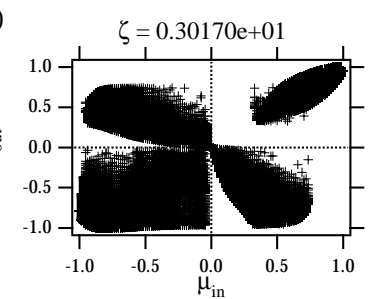

(e)

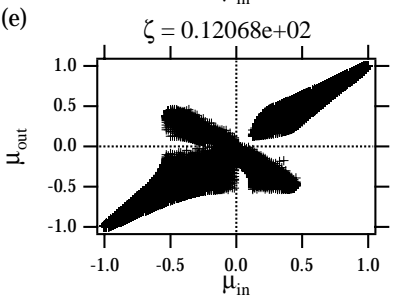

(f)

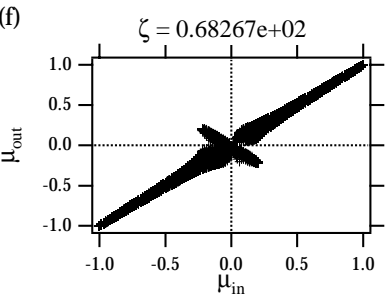

Fig. 6. Same as Fig. 2 except that the pulse winding number is $n=2$. Initial particle pitch angle cosine ranges from $\mu=-1$ to $\mu=1$, i.e., the plots represent particles injected from either side of the pulse.

This is more clearly represented in Fig. 8. The enhancement of the probability, as seen when $n=0$ and $b=6$ (Fig. 5a), appears only when the particles can resonate with the waves. On the other hand, as for the non-resonant particles, the enhancement of probability is in the adiabatic domain.

The pitch angle integrated reflection probability (corresponding to Fig. 8) is shown in Fig. 9. The reflection probability is close to the prediction of adiabatic theory when $\zeta$ is small, and it decreases with power law with large $\zeta$ asymptotically. Here the index of the power law is again $-1 / 2$. The numerical runs with other winding numbers and the wave amplitude show qualitatively similar results (not shown). Furthermore, similar results (not shown) are obtained when we use $\operatorname{sech}^{2}$ type wave packet. In all cases the pitch angle integrated probability has the common characteristics; $P \sim$ const. for small $\zeta$ and $P \sim \zeta^{-1 / 2}$ for large $\zeta$.

\section{Theoretical analysis}

It is easy to physically understand that the interaction is adiabatic when $\zeta$ is small; when the particle velocity is small or the wave packet width is large, the change of the field that a particle "sees" is small. Then how about the non-adiabatic regime?

Using $\mu(=\cos \alpha$, the pitch angle cosine of the particle) and $\psi\left(=\arctan B_{y} / B_{z}-\arctan v_{y} / v_{z}\right.$, the difference between
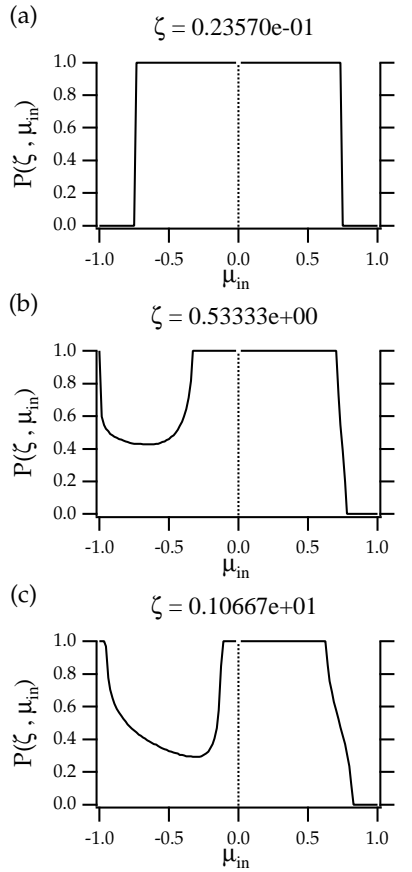

(d) $\quad \zeta=0.30170 \mathrm{e}+01$

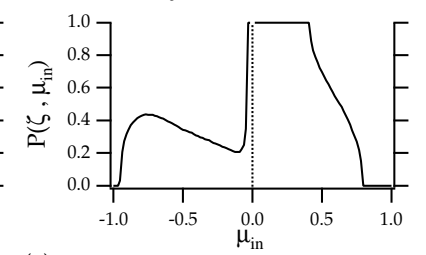

(e) $\quad \zeta=0.12068 \mathrm{e}+02$

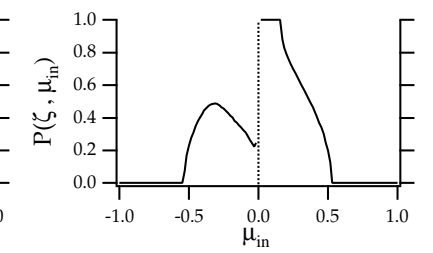

(f)

$\zeta=0.68267 \mathrm{e}+02$

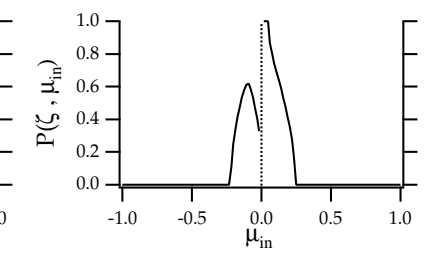

Fig. 7. Same as Fig. 3 except that the pulse winding number is $n=2$.

particle gyrophase and a wave phase), we can rewrite Eq. (3) as follows (cf, Kuramitsu and Krasnoselskikh, 2005a,b),

$\frac{d \mu}{d t}=\left(1-\mu^{2}\right)^{1 / 2} b_{w} \sin \psi$
$\frac{d \psi}{d t}=k \mu+1-\frac{\mu}{\left(1-\mu^{2}\right)^{1 / 2}} b_{w}(x) \cos \psi$,

where $b_{w}(x)$ gives the wave envelope,

$b_{w}(x)=b \mathrm{e}^{-\left(\frac{x}{l}\right)^{2}}$.

Equations (5a), (5b), and the following equation make a closed set of equations,

$\frac{d x}{d t}=\mu$.

Since the above equations is highly nonlinear, it is difficult to find an exact set of general solutions, therefore, we seek for an approximate solution by iteration.

The zeroth order trajectory without perturbation is $\mu=\mu_{0}$, $\psi=\left(k \mu_{0}+1\right) t+\psi_{0}$, and $x=x_{0}+\mu_{0} t$ where $x_{0}, \mu_{0}$, and $\psi_{0}$ are constants determined by the initial conditions. Let $\mu=\mu_{0}+\delta \mu$, and we integrate Eq. (5a) along the zeroth order trajectory, 

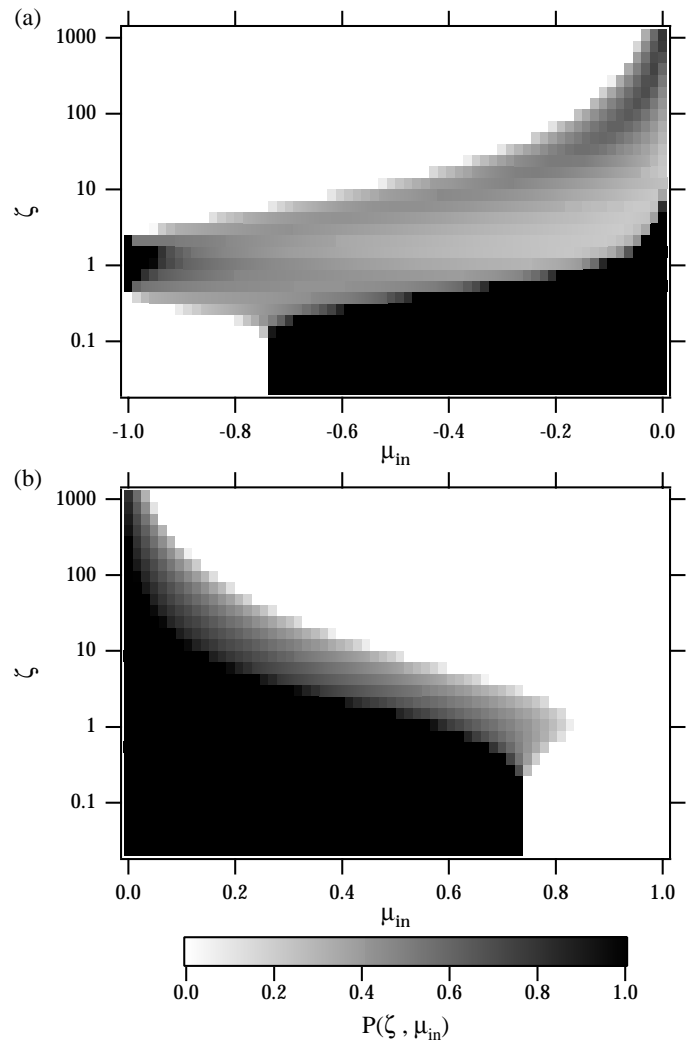

Fig. 8. Image plots of the reflection probability $P\left(\zeta, \mu_{i n}\right)$ are shown when $b=2$ and $n=2$. Panel (a) and (b) represent particles with $-1<\mu_{i n}<0$ (i.e., the particles can be cyclotron resonant with the carrier wave) and $0<\mu_{i n}<1$ (i.e., the particles cannot be cyclotron resonant with the carrier wave).

$$
\begin{aligned}
\delta \mu= & \int_{t 1}^{t 2} d t \\
& b\left(1-\mu_{0}^{2}\right)^{1 / 2} \exp \left[-\left(\frac{x_{0}+\mu_{0} t}{l}\right)\right] \sin \left(k \mu_{0} t+t+\psi_{0}\right) .
\end{aligned}
$$

Equation (8) is expressed using the error function (Akimoto, 1997).

$$
\begin{aligned}
\delta \mu= & b l \frac{\left(1-\mu_{0}^{2}\right)^{1 / 2}}{\mu_{0}} \exp \left[-\frac{1}{4}\left(n+\frac{l}{\mu_{0}}\right)^{2}\right] \\
& \times \frac{\sqrt{\pi}}{2}[\operatorname{erf}(\xi(t 2))-\operatorname{erf}(\xi(t 1))] \sin \Phi, \\
\xi(t)= & \frac{x_{0}+\mu_{0} t}{l}-\frac{i}{2}\left(n+\frac{l}{\mu_{0}}\right),
\end{aligned}
$$

where $\Phi=\psi_{0}-k x_{0}-x_{0} / \mu_{0}$ is another constant determined by the initial conditions. The first term of Eq. (9) has a factor of $\left(1-\mu_{0}^{2}\right)^{1 / 2}$, representing that beam particles $(\mu= \pm 1)$ are not influenced by the wave packet. Furthermore, the index part of Eq. (9) is more interesting; the zero point of the argument of the exponential function corresponds to the linear cyclotron resonance condition, which is written as below in original variables in the plasma frame,

$\omega-k v_{x}-\Omega=0$.

Therefore, the particles satisfying the linear resonance condition undergo strong scattering. This solution is correct within a short time scale, in which a particle begins to interact with the wave packet.

We consider whether a particle can be reflected after a long time. Although the deviation from the real trajectory grows, we can still discuss whether a particle will be reflected after a long time in the following way. By letting $t 2=-t 1=\infty$ in Eq. (9), we have,

$\mu_{\text {out }}=\mu_{0}+\sqrt{\pi} b l \frac{\left(1-\mu_{0}^{2}\right)^{1 / 2}}{\mu_{0}} \exp \left[-\frac{1}{4}\left(n+\frac{l}{\mu_{0}}\right)^{2}\right] \sin \Phi$.

A necessary condition for the particle to be reflected by the wave packet is $\mu_{\text {out }}<0(>0)$ for $\mu_{0}>0(<0)$. The ratio that $\mu_{\text {out }}$ changes the sign for particles with various gyrophase $(0<\Phi<2 \pi)$ with a certain $\mu_{0}$ gives the reflection probability $P\left(\zeta, \mu_{0}\right)$.

$$
\begin{aligned}
P\left(\zeta, \mu_{0}\right) & =\frac{2 \int_{-\pi / 2}^{\Phi_{c}} d \Phi}{\int_{0}^{2 \pi} d \Phi} \\
& =\frac{\Phi_{c}}{\pi}+\frac{1}{2}
\end{aligned}
$$

where $\Phi_{c}$ is the value of $\Phi$ at $\mu_{\text {out }}=0$, which is written as

$\Phi_{c}=-\arcsin \left\{\frac{\mu_{0}^{2}}{\sqrt{\pi} b l\left(1-\mu_{0}^{2}\right)^{1 / 2}} \exp \left[\frac{1}{4}\left(n+\frac{l}{\mu_{0}}\right)^{2}\right]\right\}$.

Since the maximum value of $\Phi_{c}$ is 0 , the reflection probability $P\left(\zeta, \mu_{0}\right)$ in Eq. (12) is $1 / 2$ at most.

This is plotted in the $\mu_{0}-\zeta$ phase space in Fig. 10. In all the plots, $b=2$, and (a) $n=0$, (b) $n=2$ resonant, and (c) $n=2$ non-resonant. The theoretical values can explain the numerically obtained reflection probability (Fig. $4 \mathrm{a}$ and 8), especially when $n=0$, except that the probability is estimated 
slightly smaller. During the adiabatic mirror reflection, the pitch angle changes the sign while conserving the energy. Therefore, it is necessary to include all the higher order terms in Eqs. (5a) and (5b). In our formulations the adiabatic mirror reflection is not treated. When $n \neq 0$, we can understand the qualitative behavior of the probability function such as the breakdown of the symmetry by the effect of the linear cyclotron resonance. When the resonance exists, the scattering is strong in the region where the index of Eq. (9) is close to 0 . When the resonance does not exist, the index will not become 0 , thus the scattering is small. The reflection probability Eq. (12), which is obtained theoretically, explains the dependence on the winding number.

We consider the power law type decay of the pitch angle integrated reflection probability in the non-adiabatic regime. When $\zeta \gg 1$, only particles with pitch angle not close to 0 or $\pi$ can be reflected by the wave packet $\left(\left|\mu_{0}\right| \ll 1\right)$, and the interaction time between the particle and the wave packet $\left(L / \mu_{0} V\right)$ is much shorter than the Larmor period $\left(\left|\mu_{0}\right| / l \gg 1\right)$. Then Eq. (11) can be rewritten as $\mu_{\text {out }} \sim \mu_{0}+$ $\sqrt{\pi} b l \mu_{0}^{-1} \mathrm{e}^{-n^{2} / 4} \sin \Phi$. When $n=0$, a necessary condition for the particle to be reflected by the wave packet is rewritten as follows.

$\mu_{0}^{2}<-\sqrt{\pi} b l \sin \Phi$

Above argument is appropriate since the upper boundary on which the reflection probability becomes 0 is written as $\mu_{0} \propto \zeta^{-1 / 2}$ in Fig. 10a. On the other hand, the lower boundary representing the adiabatic limit is expressed as $\mu_{0} \propto \zeta^{-1}$. The latter relation represents the ratio between gyroperiod and the interaction time between the particle and the wave packet. When $\mu_{0} \zeta=V \mu_{0} / L \Omega \ll 1$, i.e., when $\mu_{0} \ll 1$, even if $\zeta>1$, the interaction is still adiabatic. For $\mu_{0}<0$, letting integration domain to $-(\sqrt{\pi} b l)^{1 / 2}<\mu_{0}<0$ from Eq. (14) (because of $-1<\sin \Phi<1$ ), the pitch angle integrated reflection probability is written in the non-adiabatic limit as,

$$
\begin{aligned}
P(\zeta) & \sim \frac{\int_{-(\sqrt{\pi} b l)^{1 / 2}}^{0} P\left(\zeta, \mu_{0}\right) d \mu_{0}}{\int_{-1}^{0} d \mu_{0}} \\
& \sim \int_{-(\sqrt{\pi} b l)^{1 / 2}}^{0}\left(\frac{-1}{\pi} \arcsin \left(\frac{\mu_{0}^{2}}{\sqrt{\pi} b l}\right)+\frac{1}{2}\right) d \mu_{0} \\
& =2 \pi^{-3 / 4}(b l)^{1 / 2} \mathrm{E}\left(\frac{\pi}{4}, 2\right)
\end{aligned}
$$

where $\mathrm{E}$ is the elliptic function of the second kind. One can get the same result for $\mu_{0}>0$. It is shown that $P(\zeta)$ is proportional to $\zeta^{-1 / 2}$ as the asymptotic behavior in the nonadiabatic limit. When $n=0, P(\zeta) \sim 0.50774 \times \sqrt{b} \zeta^{-1 / 2}$. This is shown in Fig. 4b and in Fig. 5b. The analytical estimations explain the numerical results well. When $n \neq 0$ we simply

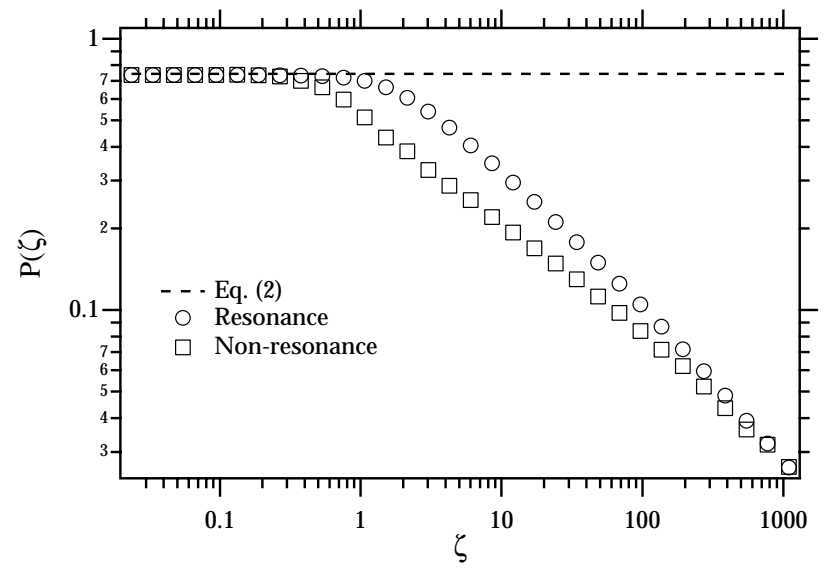

Fig. 9. Same as Fig. $4 \mathrm{~b}$ except that $n=2$. Circles and squares denote results from numerical simulation with initial particles ranging within $0<\mu_{i n}<1$ and $-1<\mu_{i n}<0$, respectively. The dotted line corresponds to the value expected from the adiabatic theory, Eq. (2).

need to multiply the estimation above by $\mathrm{e}^{-n^{2} / 8}$. The reflection probability from numerical experiments with $n=10$ (not shown) are smaller than the cases of $n=0$ and $n=2$. The above discussion is correct qualitatively, but not quantitatively. The reason of the underestimation comes from the approximation on non-adiabaticity, which is not satisfied around $\mu_{0} \sim 0$.

\section{Conclusions}

We have discussed the interaction between a charged particle and an MHD wave packet numerically and theoretically.

When the scale of the particle is smaller than the wave packet $\zeta(\equiv V / L \Omega) \ll 1$, the reflection can be well explained by the adiabatic theory. The transition from reflection to transmission is well described by the critical pitch angle; the phase integrated probability function $P\left(\zeta, \mu_{i n}\right)$ is unity or zero depending on whether the particles are outside or inside of the loss cone. The pitch angle integrated reflection probability function $P(\zeta)$ agrees with the prediction of adiabtic theory Eq. (2) when $\zeta \ll 1$. When the particle scale is comparable to the wave packet width $\zeta \sim 1$, non-adiabaticity appears. The first adiabatic invariant does not conserve any more and the reflection probability can be non-zero in the loss cone. The probability function $P(\zeta)$ decreases as the particle scale increases and approaches a asymptotic line $\zeta^{-1 / 2}$

We calculate the probability functions $P\left(\zeta, \mu_{i n}\right)$ analytically and show that the cyclotron resonance between the particle and the wave packet can explain non-adiabatic mirror reflection and the breakdown of symmetry regarding the injection pitch angle cosine, $\mu_{i n}$. The reason why the particles in resonant sense greatly deviate from the adiabatic theory is 

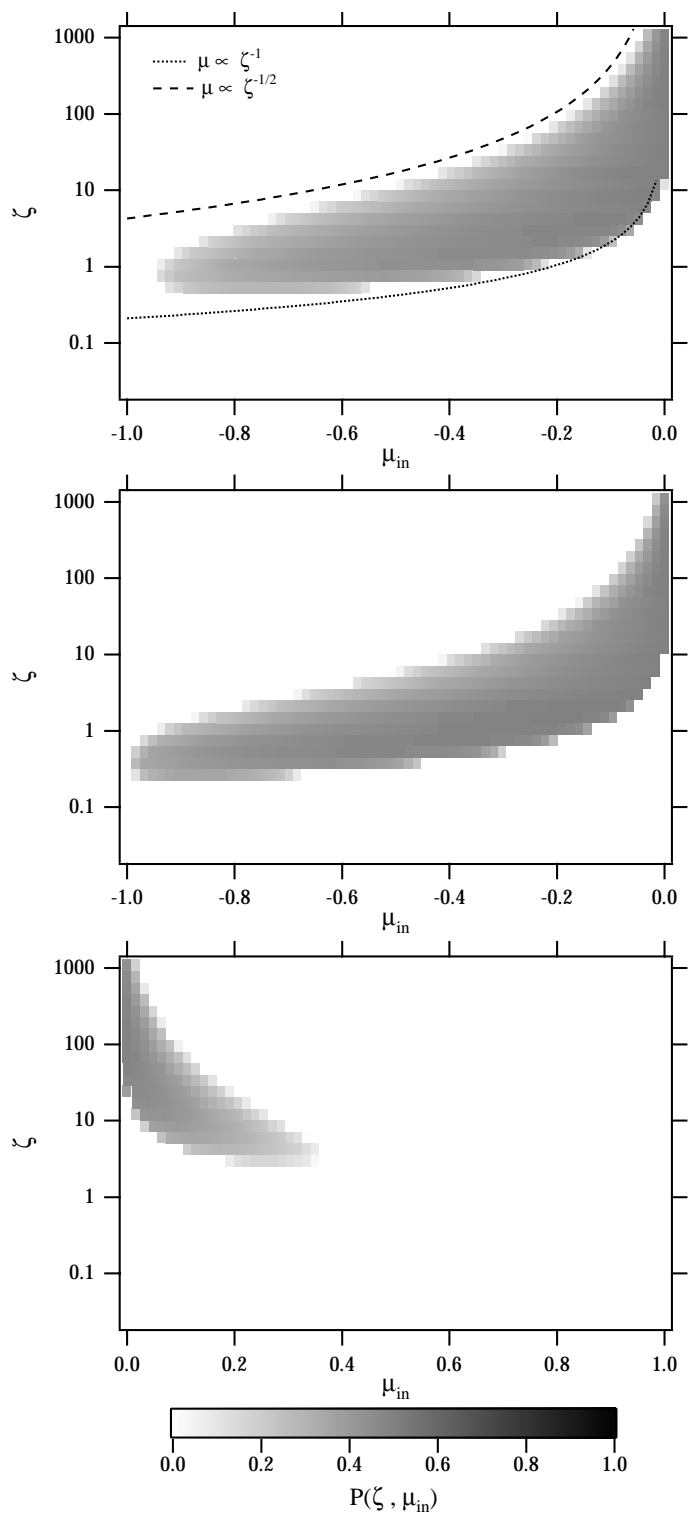

Fig. 10. Contour plots of the analytically obtained reflection probability function $P\left(\zeta, \mu_{i n}\right)$. The pulse amplitude is $b=2$, and the pulse winding number is $n=0$ (a), $n=2$ with resonant sense of polarity (b), and $n=2$ with non-resonant sense of polarity (c).

that those particles can resonant with the inner structure of wave packet. The reflection probability $P\left(\zeta, \mu_{i n}\right)$ estimated by Eq. (12) explains the reflection probability obtained by the numerical experiment.

Integration of Eq. (12) over $\mu_{0}$ gives the pitch angle integrated reflection probability function $P(\zeta)$. The assimptotic value of this function tends to be $\zeta^{-1 / 2}$. Note that the pitch angle used in this paper is in the wave packet frame, rather than, e.g., a plasma bulk frame.

The power law index $-1 / 2$ shows universality; the power law index is $-1 / 2$ independent of winding number, wave am- plitude, and whether or not particles can resonant with waves. Even when we apply the other type of wave packets such as $\operatorname{sech}^{2}$, the reflection probability function decrease as $\zeta^{-1 / 2}$.

Acknowledgements. The authors would like to thank A. Kageyama, V. Krasnoselskikh and V. Lobzin for their helpful comments.

Edited by: R. Grimshaw

Reviewed by: D. H. Burgess and another anonymous referee

\section{References}

Akimoto, K.: Theory of pulse-particle interaction in one dimension, Phys. Plasmas, 4, 3101-3112, 1997.

Classen, H.-T. and Mann, G.: Motion of ions reflected off quasiparallel shock waves in the presence of large-amplitude magnetic field fluctuations, Astron. Astrophys., 330, 381-388, 1998.

Gary, S. P. and Feldman, W. C.: A second-order theory for k parallel B sub 0 electromagnetic instabilities, Phys. Fluids, 21, 72-80, 1978.

Giacalone, J., Schwartz, S. J., and Burgess, D.: Observations of suprathermal ions in association with SLAMS, Geophys. Res. Lett., 20, 149-152, 1993.

Hada, T., Koga, D., and Yamamoto, E.: Phase coherence of MHD waves in the solar wind, Space Sci. Rev., 107, 463-466, doi: 10.1023/A:1025506124402, 2003.

Hoppe, M. M., Russell, C. T., Frank, L. A., Eastman, T. E., and Greenstadt, E. W.: Upstream hydromagnetic waves and their association with backstreaming ion populations-ISEE 1 and 2 observations, J. Geophys. Res., 86, 4471-4492, 1981.

Kennel, C. F. and Engelmann, F.: Velocity space diffusion from weak plasma turbulence in a magnetic field, Phys. Fluids, 9, 2377, 1966.

Koga, D. and Hada, T.: Phase coherence of foreshock MHD waves: wavelet analysis, Space Sci. Rev., 107, 495-498, doi:10.1023/A: 1025510225311, 2003.

Kuramitsu, Y. and Hada, T.: Acceleration of charged particles by large amplitude MHD waves: effect of wave spatial correlation, Geophys. Res. Lett., 27, 5, 629-632,, 2000.

Kuramitsu, Y. and Krasnoselskikh, V.: Acceleration of charged particles by gyroresonant surfing at quasi-parallel shocks, Astron. Astrophys., 438, 391, 2005a.

Kuramitsu, Y. and Krasnoselskikh, V.: Particle acceleration by elliptically and linearly polarized waves in the vicinity of quasiparallel shocks, J. Geophys. Res. (Space Physics), 110, A10108, doi:10.1029/2005JA011048, 2005b.

Kuramitsu, Y. and Krasnoselskikh, V.: Gyroresonant surfing acceleration, Phys. Rev. Lett., 94, 31 102, 2005c.

Lee, M. A.: Self-consistent kinetic equations and the evolution of a relativistic plasma in an ambient magnetic field, Plasma Physics, 13, 1079-1098, 1971.

Lee, M. A.: Coupled hydromagnetic wave excitation and ion acceleration upstream of the earth's bow shock, J. Geophys. Res., 87, 5063-5080, 1982.

Lucek, E., Horbury, T., Balogh, A., Dandouras, I., and Rème, H.: Cluster observations of structures at quasi-parallel bow shocks, Ann. Geophys., 22, 2309-2313, 2004, http://www.ann-geophys.net/22/2309/2004/. 
Lyons, L. R.: General relations for resonant particle diffusion in pitch angle and energy, J. Plasma Phys., 12, 45, 1974.

Mann, G. and Classen, H.-T.: Electron acceleration to high energies at quasi-parallel shock waves in the solar corona., Astron. Astrophys., 304, 576 pp., 1995.

Mann, G., Luehr, H., and Baumjohann, W.: Statistical analysis of short large-amplitude magnetic field structures in the vicinity of the quasi-parallel bow shock, J. Geophys. Res., 99, 13 315, 1994.

Nariyuki, Y. and Hada, T.: Self-generation of phase coherence in parallel Alfvén turbulence, Earth Planets Space, 57, 2005.

Nariyuki, Y. and Hada, T.: Remarks on nonlinear relation among phases and frequencies in modulational instabilities of parallel propagating Alfvén waves, Nonlin. Processes Geophys., 13, 425-441, 2006,

http://www.nonlin-processes-geophys.net/13/425/2006/.

Schlickeiser, R.: Cosmic-ray transport and acceleration. I - Derivation of the kinetic equation and application to cosmic rays in static cold media. II - Cosmic rays in moving cold media with application to diffusive shock wave acceleration, The Astrophysical Journal, 336, 243-293, 1989.
Schwartz, S. J. and Burgess, D.: Quasi-parallel shocks - A patchwork of three-dimensional structures, Geophys. Res. Lett., 18, 373-376, 1991.

Schwartz, S. J., Burgess, D., Wilkinson, W. P., Kessel, R. L., Dunlop, M., and Luehr, H.: Observations of short large-amplitude magnetic structures at a quasi-parallel shock, Geophys. Res. Lett., 97, 4209-4227, 1992.

Tsurutani, B. T., Smith, E. J., Matsumoto, H., Brinca, A. L., and Omidi, N.: Highly nonlinear magnetic pulses at Comet Giacobini-Zinner, Geophys. Res. Lett., 17, 757-760, 1990.

Tsurutani, B. T., Lakhina, G. S., Neubauer, F. M., and Glassmeier, K.: A new look at the nature of comet Halley's LF electromagnetic waves: Giotto observations, Geophys. Res. Lett., 24, 31293132, 1997. 